\title{
Mesure des débits splanchniques par dilution de marqueur : comparaison de quatre méthodes de dosage de l'acide para-amino-hippurique
}

\author{
Agnès Isserty ${ }^{\mathrm{a} *}$, Isabelle Ortigues ${ }^{\mathrm{a}}$, Didier Remond ${ }^{\mathrm{b}}$ \\ ${ }^{a}$ Laboratoire Croissance et métabolismes des herbivores, Inra, Theix, \\ 63122 Saint-Genès-Champanelle, France \\ ${ }^{b}$ Station de recherches sur la nutrition des herbivores, Inra, Theix, \\ 63122 Saint-Genès-Champanelle, France
}

(Reçu le 27 juin 1997 ; accepté le 9 janvier 1998)

\begin{abstract}
Measurement of splanchnic blood flows by marker dilution: comparison of four analytical methods of para-amino-hippuric acid. The objective of this study was to determine the influence of the analytical method of para aminohippuric acid (PAH) on splanchnic blood flows, as measured by a dye dilution method in ovines. Four different analytical methods of PAH were compared. They differed in the pretreatment of the samples and in the presence or absence of a deacetylation step to account for the partial acetylation of PAH in the liver (13\%). The optimum conditions of deacetylation were defined as $60 \mathrm{~min}$ of incubation at $90^{\circ} \mathrm{C}$ in presence of $\mathrm{HCl}(5 \mathrm{~N})$. The four analytical methods were checked to be linear (from 0 to $35 \mathrm{mg} / \mathrm{L}$ ), repeatable $(\mathrm{CV}=0.70 \%)$ and reproducible $(\mathrm{CV}=2.1 \%)$. It appeared necessary to prepare the standards in the same matrix as that of the samples. The choice of the analytical method was responsible for differences in PAH concentrations and in the absolute values for portal and hepatic blood flows, which could reach $21 \%$. The presence of a deacetylation step with $\mathrm{HCl}$ significantly reduced the contribution of the hepatic artery to the total hepatic blood flow (from 24 to $10 \%$ ). Consequently, this study showed that the nature of the analytical method chosen can highly influence the measurements of splanchnic nutrient fluxes. The recommended analytical method isthe one which includes a deacetylation step. (C) Inra/Elsevier, Paris
\end{abstract}

\section{blood flows / PAH / deacetylation / comparison / methods}

Résumé - L'objectif du travail était de déterminer l'influence de la méthode de dosage de l'acide para-amino-hippurique (PAH) sur les débits sanguins splanchniques, mesurés par dilution chez des ovins. Quatre méthodes de dosage du PAH différant par le prétraitement de l'échantillon sanguin et par l'inclusion ou non d'une étape de déacétylation ont été comparées, la déacétylation permettant de doser le PAH qui se trouve en partie acétylé au

\footnotetext{
* Correspondance et tirés à part
} 
niveau du foie $(13 \%)$. Les conditions optimales de déacétylation ont été définies à $60 \mathrm{~min}$ d'incubation à $90^{\circ} \mathrm{C}$ en présence d' $\mathrm{HCl} 5 \mathrm{~N}$. Les quatre méthodes de dosage se sont révélées linéaires $(0$ à $35 \mathrm{mg} / \mathrm{L})$, répétables $(\mathrm{CV}=0,70 \%)$ et reproductibles $(\mathrm{CV}=2,1 \%)$ et il paraît souhaitable de réaliser la gamme dans la même matrice que les échantillons à doser. Le choix de la méthode de dosage entraîne des différences d'estimation des concentrations en PAH et donc des valeurs absolues de débits porte et sus-hépatique pouvant atteindre $21 \%$. La déacétylation en présence d'HCl réduit significativement la contribution de l'artère hépatique au débit hépatique total (de $24 \%$ à $10 \%$ ). Par conséquent, les choix analytiques peuvent fortement influencer les résultats de la mesure des flux splanchniques de nutriments chez les ovins. La méthode retenue est celle qui inclut une étape de déacétylation. (O) Inra/Elsevier, Paris

débits sanguins / PAH / déacétylation / comparaison / méthodes

\section{INTRODUCTION}

Lors de toute étude métabolique réalisée in vivo au niveau tissulaire, la détermination des flux de nutriments afférents à et efférents de ce tissu repose sur la mesure des débits sanguins irriguant chacun des tissus et des concentrations en métabolites sanguins au niveau artériel et veineux. Or, une difficulté majeure de ce type d'étude réside dans la précision de la mesure des débits sanguins. La principale méthode utilisée actuellement au niveau splanchnique est une méthode de dilution dont le marqueur est le plus souvent l'acide para-amino-hippurique (PAH). Cette méthode est entachée d'une grande variabilité qui provient de facteurs tels que le mauvais mélange du marqueur dans le sang, le choix du site d'infusion ou un mauvais placement de cathéters [25].

Parmi les autres facteurs qui peuvent modifier les résultats de débit splanchnique se trouve la méthode de dosage du marqueur. Diverses procédures ont été utilisées. Elles diffèrent tout d'abord par le mode de déprotéinisation de l'échantillon qui est réalisée soit en milieu basique [23], acide [19, 27, 44] ou par dialyse [46]. D'autre part, lors de la mesure de débit sanguin au niveau du foie, une autre étape du dosage devient importante, elle correspond à l'inclusion $[27,44]$ ou non [ 19 ,
23, 46] d'une étape de déacétylation du PAH. En effet, d'après Katz et Bergman [27], le PAH se trouve en partie métabolisé au niveau du foie en PAH acétylé. Il n'est alors pas détectable par les méthodes qui n'incluent pas d'étape de déacétylation de l'échantillon. Ceci entraîne une sous-estimation des concentrations en PAH du sang dans la veine sus-hépatique (VSH) et donc une surestimation des débits sanguins au niveau du foie et en particulier de la contribution de l'artère hépatique $(\mathrm{AH})$ au débit hépatique total. De plus, les conditions de déacétylation differrent selon les auteurs $[27,29,44]$.

Le premier objectif de ce travail a été de déterminer les conditions optimales de déacétylation du PAH (température et temps d'incubation). Le second objectif a été de tester l'influence du mode de déprotéinisation et de la déacétylation sur la qualité des méthodes d'analyse (linéarité, répétabilité, reproductibilité et justesse). Pour cela quatre méthodes de dosage (déprotéinisation en milieu basique, par dialyse ou en milieu acide avec ou sans déacétylation) ont été comparées. Le troisième objectif était de comparer les concentrations de PAH aux niveaux artériel et veineux, la variabilité des débits sanguins et les valeurs absolues des débits splanchniques obtenues à l'aide des différentes méthodes testées, afin de mieux définir les conditions de mesure des débits 
sanguins à l'aide de PAH. Ce travail est complémentaire d'une étude réalisée pour déterminer la fiabilité de la mesure des débits sanguins en veine porte par débitmétrie ultrasonique chez des ovins [41].

\section{MATÉRIEL ET MÉTHODES}

\subsection{Animaux et alimentation}

Cinq moutons adultes, de race Texel, de poids vif (PV) initial de $54 \mathrm{~kg} \pm 5,5$ ont été utilisés. Ils ont été équipés chirurgicalement de cathéters sanguins placés au niveau de la veine porte (VP), d'une veine sus-hépatique (VSH), des veines mésentérique (VM) et ruminale (VR) et d'une artère mésentérique (AM) selon les méthodes décrites par Ortigues et al. [37] et Rémond et al. [40].

Les animaux étaient logés dans une pièce climatisée à éclairage permanent, dans des cages individuelles sur caillebotis.

Le régime était composé de foin de montagne à base de dactyle (7,5 MJ d'énergie métabolisable/ $\mathrm{kg}$ de matière sèche (MS) ; 18,3g $\mathrm{N} / \mathrm{kg}$ MS). Les moutons étaient alimentés à un niveau proche de l'entretien ( $970 \mathrm{~g} \mathrm{MS}$ de foin $/ \mathrm{j}$ ), en huit repas égaux distribués toutes les $3 \mathrm{~h}$ à l'aide de distributeurs automatiques d'aliment.

\subsection{Mesures}

Les débits sanguins en VP et VSH ont été mesurés au cours d'une perfusion de PAH simultanément en VM et en VR [37], au taux de $0,2 \mathrm{mg} \mathrm{PAH} / \mathrm{min} / \mathrm{kg}$ PV et après une surcharge de PAH $(3,75 \mathrm{mg} / \mathrm{kg} \mathrm{PV})$. Les prélèvements sanguins ont été effectués simultanément en AM, VP et VSH toutes les $20 \mathrm{~min}$ pendant $6 \mathrm{~h}$ soit deux cycles alimentaires. Deux séries de prélèvements ont été effectuées sur deux journées pour chaque animal. Malheureusement, suite à des dysfonctionnements de cathéters, seuls 144 prélèvements ont été utilisables au lieu des 180 prévus pour chaque vaisseau (AM, VP et VSH).

Les échantillons de $5 \mathrm{~mL}$ de sang chacun ont été prélevés sur EDTA et conservés à
$-20{ }^{\circ} \mathrm{C}$, afin de doser le PAH selon quatre méthodes différentes.

\subsection{Analyses}

\subsubsection{Principes}

Toutes les méthodes de dosage du PAH décrites dans cette étude ont été habituellement utilisées dans les études de métabolisme tissulaire réalisée in vivo chez les ruminants.

Le premier point de divergence des méthodes utilisées résidait dans le prétraitement de l'échantillon, et en particulier le mode de déprotéinisation de l'échantillon. Pour la méthode en « milieu basique ", un volume de sang total dilué avec un volume d'eau distillée est déprotéinisé à l'aide d'un volume de soude $0,4 \mathrm{~N}$ et d'un volume de sulfate de zinc à $4 \%$ (méthode similaire à celle décrite par [23]). Pour la méthode en « milieu acide » un volume de sang total dilué dans 2 volumes d'eau distillée est déprotéinisé à l'aide d'un volume d'acide trichloracétique à $20 \%$ [19] et pour la méthode avec "dialyse " le dosage est réalisé sur autoanalyseur sans dilution préalable [46]. La quatrième méthode a consisté à inclure une étape de déacétylation du PAH à la méthode effectuée en "milieu acide".

Les quatre méthodes de dosage du PAH sont basées sur la réaction colorée de Bratton et Marshall [5]. La fonction amine libre de l'acide PAH est diazotée par le nitrite de sodium, l'excès de nitrite étant détruit par du sulfamate d'ammonium. Le diazoïque obtenu forme un complexe coloré en présence de N-Naphthyl Éthylenediamine en milieu acide.

\subsection{2. Étude des conditions de déacétylation du PAH}

Avant de comparer les conditions analytiques de déacétylation du $\mathrm{PAH}$, une première étape a consisté à préparer du PAH acétylé d'après la méthode de Newman et al. [35]. La pureté du produit a été testée par résonance magnétique nucléaire sur spectromètre (AMX 400 Buker). Le signal du proton a été obtenu à la fréquence de $400 \mathrm{MHz}$ sur la base de 32 acquisitions. L'analyse spectrale du PAH acétylé comparée à celle du PAH (figure 1) a permis de vérifier la pureté du PAH acétylé préparé. Puis les conditions de déacétylation ont 


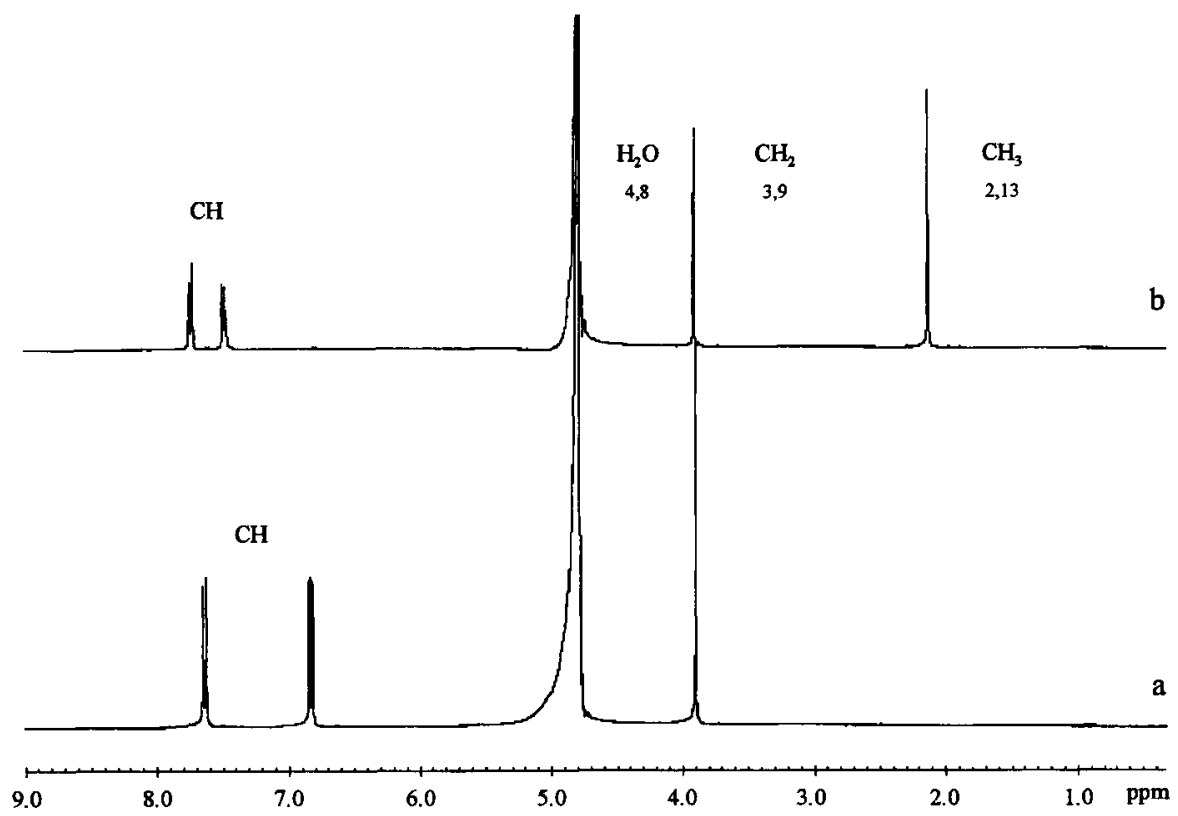

Figure 1. Analyse spectrale du PAH (a) et du PAH acétylé (b). Les signaux (ppm) du $\mathrm{CH}_{3} \mathrm{du}$ groupe acétyle, du $\mathrm{CH}_{2}$ du groupe glycine et des protons de l'eau sont indiqués.

été testées en comparant trois températures d'incubation $\left(80,90\right.$ et $\left.95^{\circ} \mathrm{C}\right)$ et cinq durées d'incubation $(15,30,60,90$ et $120 \mathrm{~min})$ en présence d' $\mathrm{HCl} 5 \mathrm{~N}$ (1 volume d' $\mathrm{HCl}$ pour 4 volumes de surnageant). Ces deux premiers paramètres ont été choisis sur la base des méthodes déjà publiées [27, 29,44]. Deux tests supplémentaires ont été réalisés : le premier à température ambiante afin de vérifier la nonhydrolyse du PAH acétylé dans ces conditions et le second a consisté à reprendre les conditions d'hydrolyse $\left(100^{\circ} \mathrm{C}\right.$ pendant $30 \mathrm{~min}$ sans ajout d' $\mathrm{HCl}$ ) citées par Katz et Bergman [27]. Ces tests ont été réalisés à partir d'une solution de PAH acétylé à $10 \mathrm{mg} / \mathrm{L}$ en solution dans de l'eau ou dans du sang.

\subsection{3. Évaluation de la qualité des méthodes de dosages}

La linéarité a été évaluée entre 0 et $35 \mathrm{mg} / \mathrm{L}$ à partir d'une solution aqueuse de PAH. Le dosage de tous les échantillons sanguins a été réalisé en double. La répétabilité et la repro- ductibilité ont été évaluées à partir d'une solution aqueuse de PAH à $10 \mathrm{mg} / \mathrm{L}$. La répétabilité a été réalisée dans une seule série d'analyses, en effectuant dix déterminations afin d'obtenir le coefficient de répétabilité. Pour la reproductibilité, le même échantillon a été dosé dans quinze séries d'analyses différentes, effectuées sur plusieurs journées impliquant à chaque fois une nouvelle préparation de gammes, afin d'obtenir un coefficient de variation (CV) de reproductibilité. La justesse a été évaluée par la méthode des surcharges à partir d'un échantillon de référence de sang total dosé en double. Pour les méthodes manuelles, les concentrations de PAH ont été obtenues en dosant les échantillons contre une gamme préparée dans le sang. Pour la méthode automatique, les concentrations de PAH ont été obtenues à partir d'une gamme préparée dans du $\mathrm{NaCl}$ à $0,9 \%$ et en soustrayant, au sein de chaque série d'analyse, la valeur du blanc échantillon. En effet, il est connu que la matrice sanguine interférait avec le dosage [21]. Les taux de récupération ont alors pu être calculés. 


\subsection{Calculs des débits sanguins}

Les débits splanchniques instantanés (VP et VSH) obtenus ont été calculés à partir des concentrations en PAH dans les vaisseaux afférents et efférents des organes et du taux de perfusion (voir encadré).

Les valeurs instantanées de débits splanchniques ont ensuite été utilisées pour effectuer les comparaisons entre méthodes.

\subsection{Analyses et traitements statistiques}

Une première analyse a permis d'évaluer pour chaque méthode les critères de qualité analytique : la linéarité, l'écart entre doubles, la répétabilité, la reproductibilité et la justesse. Ces critères ont ensuite été comparés à l'aide d'un test $t$ de Student.

Une deuxième analyse a été réalisée afin de tester l'influence des différentes méthodes de dosage du PAH sur la valeur absolue des débits splanchniques et leur variabilité. Dans ce but, les débits instantanés ont été comparés par test $t$ de Student apparié. La variabilité a été testée en comparant les $\mathrm{CV}$ associés aux débits obtenus par cycle alimentaire, sur la base de leurs intervalles de confiance calculés selon Sokal et Rohlf [45].

\section{RÉSULTATS}

\section{1. Étude des conditions de déacétylation du PAH}

L'étude des conditions de déacétylation du PAH en milieu aqueux montre que les différents facteurs testés, température, temps d'incubation et ajout d' $\mathrm{HCl}$ influencent les taux de récupération de manière significative (figure 2). Les résultats obtenus à température ambiante et sans $\mathrm{HCl}$ confirment que le PAH acétylé n'est pas hydrolysé dans ces conditions. Pour obtenir une hydrolyse complète du $\mathrm{PAH}$ acétylé, il faut être en présence d' $\mathrm{HCl}$ et incuber l'échantillon pendant un minimum de 30 min à une température minimale de $90^{\circ} \mathrm{C}$. Néanmoins, au-delà de $30 \mathrm{~min}$ d'incubation à $95^{\circ} \mathrm{C}$, la répétabilité des résultats est plus faible.

Les conditions analytiques optimales, retenues pour être testées sur sang total sont donc une température de $90^{\circ} \mathrm{C}$ et des temps d'incubation de 30,60 et $90 \mathrm{~min}$. Les tests effectués (figure 2) montrent une influence significative $(p<0,05)$ du temps d'incubation sur les taux de récupération. Seul, le taux de récupération obtenu après 60 min d'incubation n'est pas significativement différent de $100 \%$.

$$
\begin{aligned}
& \text { débits sanguins VP }(\mathrm{L} / \mathrm{min})=\frac{\text { Vitesse infusion }(\mathrm{L} / \mathrm{min}) \times \text { conc. PAH infusé }(\mathrm{mg} / \mathrm{L})}{(\text { conc. PAH VP }- \text { conc. PAH AM })(\mathrm{mg} / \mathrm{L})} \\
& \text { débits sanguins VSH }(\mathrm{L} / \mathrm{min})=\frac{\text { Vitesse infusion }(\mathrm{L} / \mathrm{min}) \times \text { conc. PAH infusé }(\mathrm{mg} / \mathrm{L})}{(\text { conc. PAH VSH }- \text { conc. PAH AM) }(\mathrm{mg} / \mathrm{L})} \\
& \text { débits sanguins AH }(\mathrm{L} / \mathrm{min})=\text { débits sanguins VSH }- \text { débits sanguins VP } \\
& \text { Contribution de AH }(\%)=\frac{\text { débits sanguins AH }(\mathrm{L} / \mathrm{min})}{\text { débits sanguins VSH }(\mathrm{L} / \mathrm{min})} \\
& \text { conc. PAH infusé = concentration de PAH dans la solution infusée } \\
& \text { conc. PAH VP = concentration de PAH en veine porte } \\
& \text { conc. PAH VSH = concentration de PAH en veine sus-hépatique } \\
& \text { conc. PAH AM = concentration de PAH en artère mésentérique }
\end{aligned}
$$




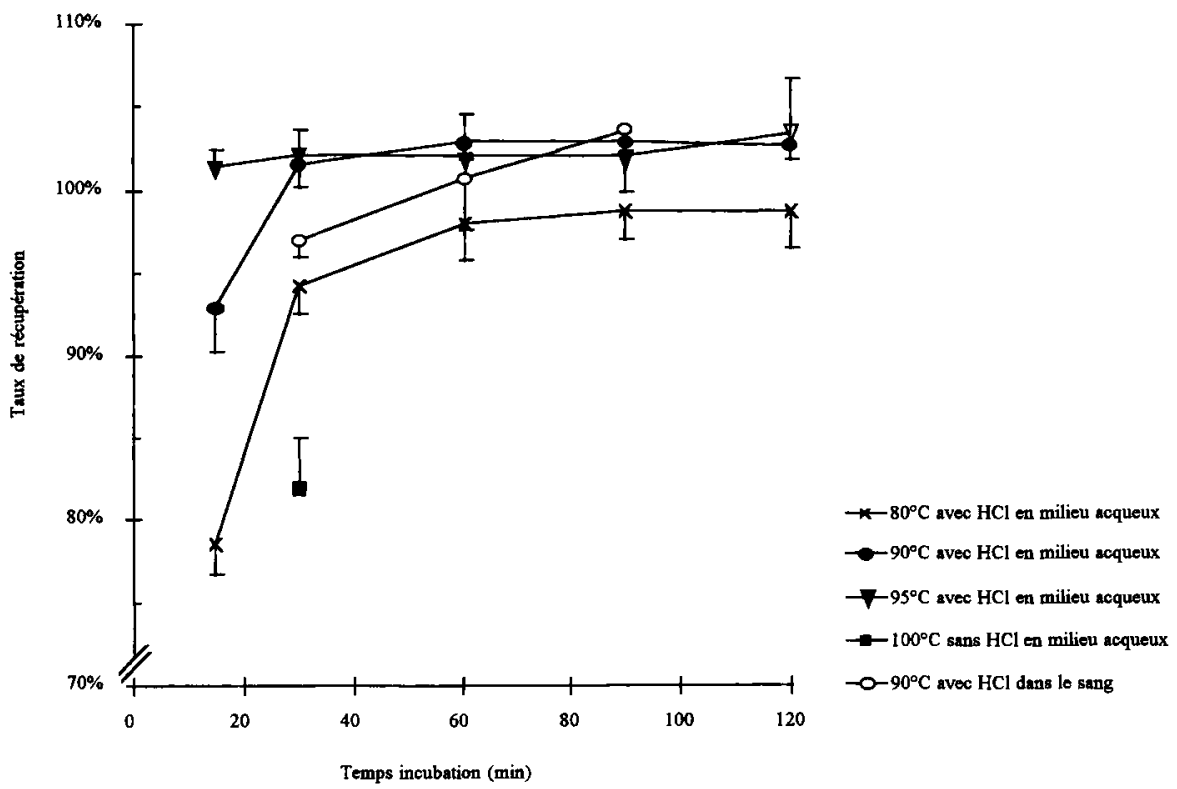

Figure 2. Influence du temps et de la température d'incubation ainsi que de la présence d' $\mathrm{HCl}$ sur les taux de récupération du PAH acétylé.

En conclusion, les conditions optimales de déacétylation retenues sont une température de $90^{\circ} \mathrm{C}$ et un temps d'incubation de $60 \mathrm{~min}$ avec ajout d' $\mathrm{HCl}$.

\section{2. Évaluation de la qualité des différentes méthodes de dosage}

Toutes les méthodes de dosage se sont révélées linéaires entre 0 et $35 \mathrm{mg} / \mathrm{L}$ avec des coefficients de corrélation compris entre 0,9997 et 0,9999 .

Les écarts entre doubles sont faibles, $1,26 \%$ en moyenne pour l'ensemble des méthodes (tableau $I$ ), avec des écarts minimaux et maximaux respectivement pour la méthode en « milieu basique » ou avec " dialyse ». La répétabilité des résultats est généralement bonne avec des $\mathrm{CV}$ compris entre 0,5 (méthode en " milieu basique $»$ ) et $0,9 \%$ (« milieu acide avec étape de déacétylation $\gg), p<0,05$ (tableau I).

La reproductibilité est aussi bonne (CV moyen de $2,1 \%$ ) sans différence significative entre les méthodes (tableau I).

Les taux de récupération se sont élevés respectivement à 101,5\% $( \pm 0,21)$, $102,1 \%( \pm 1,38), 101,0 \%( \pm 0,73)$ et $100,2 \%( \pm 0,52)$ pour les méthodes « en milieu basique », « en milieu acide sans déacétylation », « dialyse " et « en milieu acide avec déacétylation ». Ces taux de récupération ne sont pas significativement différents de $100 \%$.

\subsection{Comparaison des concentrations en PAH}

Quel que soit le compartiment sanguin considéré (A, VP et VSH), la méthode de 
Tableau I. Influence de la méthode de dosage du PAH sur les écarts entre doubles exprimés en pourcentage (moyenne \pm écart type de la moyenne) et les coefficients de variation de répétabilité et de reproductibilité.

Écarts entre double

Milieu basique

Milieu acide sans déacétylation

Dialyse

Milieu acide avec déacétylation

Répétabilité

Milieu basique

Milieu acide sans déacétylation

Dialyse

Milieu acide avec déacétylation

Reproductibilité

Milieu basique

Milieu acide sans déacétylation

Dialyse

Milieu acide avec déacétylation
Moyennes des écarts (\%)

$1,12^{\mathrm{a}} \pm 0,165$

$1,20^{\mathrm{ab}} \pm 0,056$

$1,38^{\mathrm{b}} \pm 0,082$

$1,35^{\mathrm{ab}} \pm 0,114$

Coefficient de variation (\%)

$$
\begin{gathered}
0,51^{\mathrm{a}} \\
0,64^{\mathrm{ab}} \\
0,59^{\mathrm{ab}} \\
0,91^{\mathrm{b}}
\end{gathered}
$$

Coefficient de variation (\%)

$1,93^{\mathrm{a}}$

$1,89^{\mathrm{a}}$

$2,28^{\mathrm{a}}$

$2,23^{\mathrm{a}}$

a, b, c Pour chaque paramètre, les valeurs présentant des exposants différents, sont significativement différentes $(p<0,05)$.

dosage influe de manière significative sur les concentrations en PAH (tableau II). La méthode en " milieu basique » donne les concentrations en PAH les plus faibles alors que la méthode en « milieu acide sans déacétylation » donne des résultats supérieurs d'environ de $2,6 \%$ en $A, 6,9 \%$ en VP et $7,5 \%$ en VSH $(p<0,05)$. Cet écart est encore plus important avec la méthode avec « dialyse » pour laquelle les concentrations en PAH obtenues sont supérieures à la méthode en « milieu basique » de 6,1 $\%$ en $\mathrm{A}, 11,7 \%$ en VP et $11,5 \%$ en VSH $(p<0,05)$. Il est intéressant de noter que les différentes méthodes induisent aussi des écarts différents entre compartiments sanguins, ainsi la différence VP-A est respectivement de 4,6, 5,4,5,8 $\mathrm{mg} / \mathrm{L}$ avec les méthodes en « milieu basique », en « milieu acide sans déacétylation " et avec " dialyse ». Par contre, la différence VPVSH est beaucoup moins variable entre ces trois méthodes.
Si on compare la méthode en « milieu acide avec déacétylation » à la méthode en « milieu acide sans déacétylation », on observe une augmentation significative $(p$ $<0,05$ ) de l'estimation des concentrations de $1,16 \mathrm{mg} / \mathrm{L}$ soit $10 \%$ au niveau artériel, de $0,92 \mathrm{mg} / \mathrm{L}$ soit $5 \%$ au niveau porte et de $1,5 \mathrm{mg} / \mathrm{L}$ soit $10 \%$ au niveau sus-hépatique.

\subsection{Comparaison des valeurs absolues de débits sanguins}

Au niveau de la veine porte (tableau II), les méthodes en « milieu acide avec ou sans déacétylation » donnent des débits sanguins similaires (différences de $0,04 \mathrm{~L} / \mathrm{min}$ (NS)). Les débits sanguins mesurés par l'intermédiaire de la méthode avec « dialyse » sont légèrement inférieurs à ceux obtenus avec la méthode en « milieu acide sans déacétylation » (dif- 
A. Isserty et al.

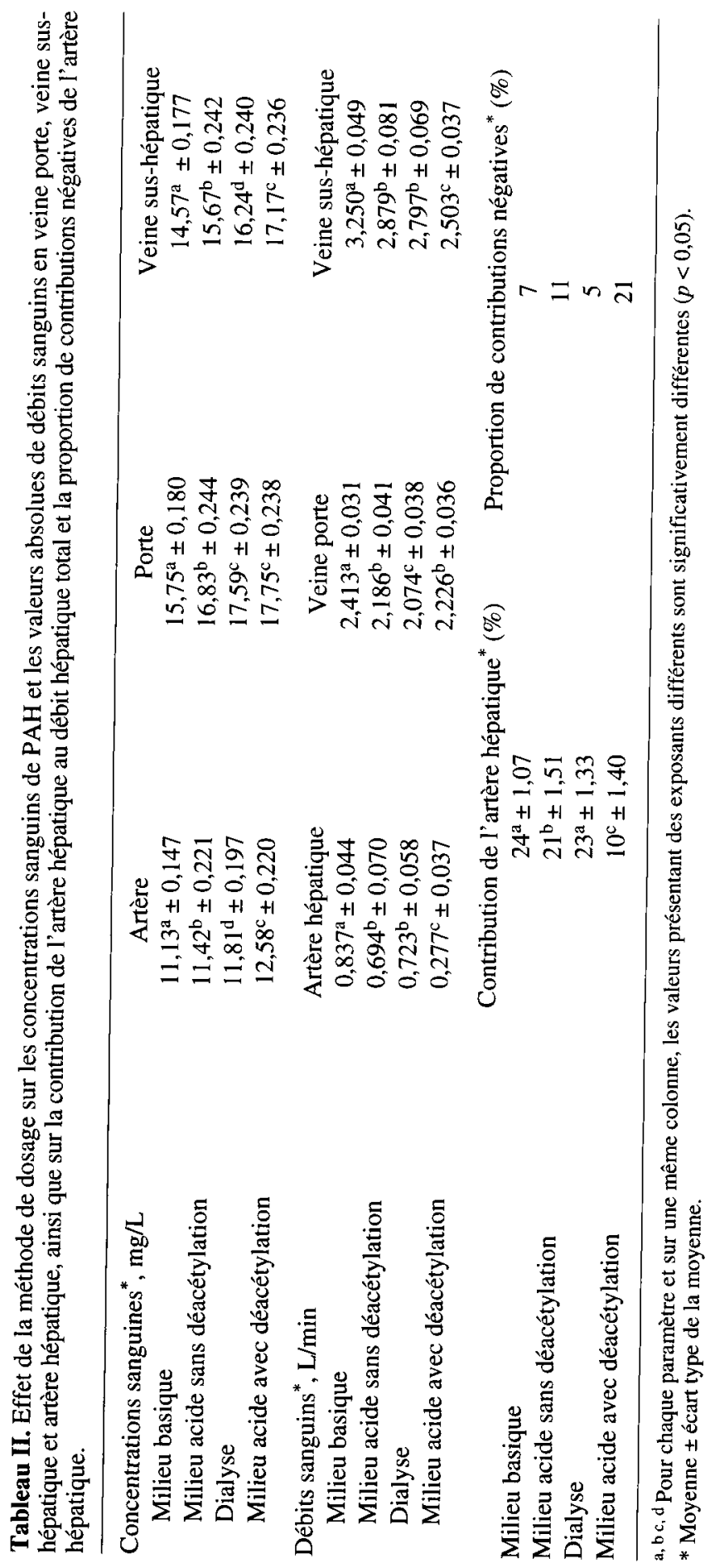


férences de $-5 \%, p<0,05)$. La méthode en " milieu basique » donne les débits sanguins les plus élevés. Ils sont en moyenne supérieurs de $10 \%$ à ceux obtenus avec la méthode en « milieu acide sans déacétylation » et de $16 \%$ par rapport à la méthode avec « dialyse » $(p<0,05)$.

Au niveau de la veine sus-hépatique et de l'artère hépatique (tableau $I I$ ), les méthodes en « milieu acide sans déacétylation » et avec « dialyse » donnent des résultats similaires. Par rapport à ces deux méthodes, les débits sanguins obtenus avec la méthode en « milieu basique » sont en moyenne supérieurs de $15 \%$ pour la VSH et de $18 \%$ pour l'AH $(p<0,05)$. Comparée à la méthode en «milieu acide sans déacétylation » la méthode en « milieu acide avec déacétylation » conduit à des valeurs de débits sanguins inférieures de $0,376 \mathrm{~L} / \mathrm{min}$ soit $-15 \%$ en VSH et de $0,417 \mathrm{~L} / \mathrm{min}$ soit $-150 \%$ en $\mathrm{AH}$.

La contribution de l'artère hépatique au débit hépatique total (tableau II) est en moyenne de $23 \%$ pour les trois méthodes sans étape de déacétylation et de $10 \%$ pour la méthode en « milieu acide avec déacétylation », celle-ci est significativement différente des trois autres méthodes $(p<0,05)$. Toutefois, la méthode en « milieu acide avec déacétylation » fait apparaître un plus grand pourcentage de contributions négatives ( $21 \%$ ) au lieu de 5 à $11 \%$ pour les autres méthodes.

\subsection{Comparaison de la variabilité des débits sanguins}

Les CV associés aux débits moyens obtenus par cycle alimentaire (tableau III) étaient en moyenne de $15 \%$ en VP, de $14 \%$ en VSH et de $76 \%$ en $\mathrm{AH}$, sans différence significative entre méthodes de dosage.

\section{DISCUSSION}

\subsection{Conditions de déacétylation}

Chez le mouton, une partie du PAH infusé se trouve acétylée au niveau du foie [27]. Il est donc nécessaire de doser le PAH par une méthode qui inclut une étape de déacétylation lorsque l'on désire mesurer les débits splanchniques totaux. Dans le but de tester les conditions de déacétylation, nous avons dans un premier temps préparé du PAH acétylé selon Newman [35]. La pureté du produit obtenu a été vérifiée par RMN ; elle s'est avérée bonne, ce qui nous a conduit, à la deuxième étape qui était de tester les conditions optimales de déacétylation. En effet, plusieurs auteurs ont déjà appliqué des méthodes de déacétylation qui diffèrent par la température et le temps d'incubation choisis ainsi que par la présence ou non d' $\mathrm{HCl}$ $[11,27,28,29,32,44]$. Tout d'abord,

Tableau III. Influence de la méthode de dosage du PAH sur les coefficients de variation moyens (moyenne en $\% \pm$ écart type de la moyenne) des débits sanguins obtenus par cycle alimentaire ( $n=16$ cycles alimentaires).

\begin{tabular}{lccc}
\hline & \multicolumn{3}{c}{ CV moyen / cycle alimentaire (\%) } \\
& Artère hépatique & Veine porte & Veine sus-hépatique \\
\hline Milieu basique & $55,67 \pm 6,34$ & $15,54 \pm 0,54$ & $13,29 \pm 1,01$ \\
Milieu acide sans déacétylation & $89,58 \pm 17,84$ & $14,62 \pm 1,89$ & $14,10 \pm 1,79$ \\
Dialyse & $67,89 \pm 11,69$ & $13,97 \pm 1,65$ & $15,90 \pm 2,83$ \\
Milieu acide avec déacétylation & $89,89 \pm 43,85$ & $15,10 \pm 1,61$ & $11,68 \pm 1,05$ \\
\hline
\end{tabular}


l'évaluation des conditions de déacétylation montre une moins bonne répétabilité lorsque la température et le temps d'incubation augmentent. Cela est probablement dû à une évaporation du surnageant dans les cupules utilisées. Ce problème d'évaporation a déjà été soulevé par d'autres auteurs $[22,39]$, ce qui a conduit Herdt et al., [22] à modifier la méthode de Katz et Bergman [27], en effectuant la déacétylation dans des cupules scellées. En ce qui concerne le taux de récupération, Katz et Bergman [27] ont obtenu des valeurs comprises entre 98 et $100 \%$ après une incubation de $30 \min$ à $100^{\circ} \mathrm{C}$, alors que notre résultat n'est que de $82 \%$. Dans nos conditions, il s'est avéré que la présence d' $\mathrm{HCl}$ était essentielle pour une déacétylation complète et reproductible du PAH. L'ajout d' $\mathrm{HCl}$ est préconisé par Kirch et Bergeim [28], Smith et al. [44], Looyé [32], Brown et al. [11] et abandonné par Katz et Bergman [27]. L'acide chlorhydrique limiterait la dégradation du TCA et donc le dégagement de $\mathrm{CO}_{2}$ et la formation de chloroforme [22]. Suite à nos essais nous recommandons une incubation à $90^{\circ} \mathrm{C}$ pendant $60 \mathrm{~min}$, ce qui permet une récupération de $100 \%$ accompagnée d'un écart type de la moyenne relativement faible.

\section{2. Évaluation de la qualité des différentes méthodes de dosage}

La qualité des quatre méthodes de dosage s'est globalement avérée satisfaisante en termes de linéarité, de répétabilité et de reproductibilité. Les deux derniers critères étaient en accord avec les CV intra et inter-essais obtenus par Wray-Cahen et al. [48] qui étaient inférieurs à $3 \%$. Sur les bases de ces critères, les conditions d'utilisation de cette méthode de mesure des débits sanguins splanchniques peuvent être précisées. En effet, un coefficient de répétabilité moyen de $0,7 \%$ per- met de détecter des différences entre concentrations de PAH de l'ordre de 0,7 à $1,6 \%$ intra-journée ; les débits sanguins instantanés sont alors connus avec une erreur maximale de $4 \%$. Un coefficient de reproductibilité moyen de $2,1 \%$ permet de détecter une différence de concentrations de PAH de l'ordre de 2,8 à 3,3\% inter-journée, ce qui conduit à une erreur maximale de $15 \%$ au niveau des débits sanguins déterminés sur des jours différents. Cette plage d'incertitude est importante à considérer lors de comparaisons statistiques entre traitements expérimentaux sur animaux, en particulier dans le cas des débits $\mathrm{AH}$.

Les tests de justesse ont aussi été satisfaisants pour toutes les méthodes, sachant que les précautions avaient été prises de s'affranchir d'un effet matrice. Harvey et Brothers [21] avaient montré l'obtention d'une coloration avec des «blancs » plasmatiques et sanguins et avaient testé l'influence de certains métabolites sanguins (glucose et urée) sur les concentrations de PAH sans en mesurer d'interférence. En conséquence, il semble souhaitable d'effectuer une gamme dans du sang pour effacer les effets matrices.

\subsection{Comparaison des concentrations de PAH}

Les résultats présents montrent que le mode de déprotéinisation de l'échantillon influence les concentrations de $\mathrm{PAH}$, ainsi que l'avait déjà constaté Looyé [32]. Néanmoins, les écarts obtenus entre les trois méthodes de dosage sans étape de déacétylation ne peuvent pas s'expliquer sur la base des taux de récupération dans nos conditions de dosage (gamme dans le sang).

L'inclusion d'une étape de déacétylation en milieu acide provoque une augmentation des concentrations en artère et en veine porte similaire, de l'ordre de 
$1 \mathrm{mg} / \mathrm{L}$ et en VSH de l'ordre de $1,5 \mathrm{mg} / \mathrm{L}$, alors que Katz et Bergman [27] avaient montré des augmentations respectives de $1,3 \mathrm{mg} / \mathrm{L}$ et $1,9 \mathrm{mg} / \mathrm{L}$ pour un même niveau d'infusion de PAH. Dans les deux cas la variabilité inter-animaux étaient importante $(\mathrm{CV}=90 \%)$. Ces résultats indiquent qu'en moyenne $13 \%$ du PAH infusé est acétylé au niveau du foie.

\subsection{Comparaison des valeurs absolues de débits sanguins}

La présente étude montre que les valeurs de débits sanguins varient suivant la méthode de dosage employée, et qu'il est important de tenir compte des aspects méthodologiques pour comparer des résultats bibliographiques entre eux. En l'absence de comparaison des débits sanguins avec des valeurs de référence, le choix de la méthode optimale doit se baser sur des critères indirects. Ainsi dans un premier temps, sur la base de résultats bibliographiques obtenus sur ovins (tirés en partie de Ortigues et Visseiche [36]), des régressions ont été établies, pour chaque méthode de dosage utilisée, entre les débits sanguins porte et sus-hépatique et l'énergie métabolisable ingérée. Ces régressions entachées d'une forte variabilité n'ont pas permis de conclusion claire. En revanche les contributions de l'artère hépatique au débit hépatique total semblent constituer un critère discriminatoire.

Dans notre expérience, ces contributions sont de l'ordre de $20 \%$ pour les méthodes de dosage effectuées sans étape de déacétylation. Ces résultats sont en accord avec ceux de six publications sur ovins [14-18, 38] pour lesquelles le dosage de PAH a été réalisé avec la méthode « dialyse ». La déacétylation en présence d' $\mathrm{HCl}$ fait apparaître une contribution de l'ordre de $10 \%$ en accord avec les contributions publiées par Lobley [29,
30], et Lobley et al. [31] qui se situent entre 2 et $10 \%$, et celles obtenues sur ovins par injection de microsphères 6 à $10 \%[1,2]$. Cette dernière méthode peut être considérée comme une méthode de référence pour la mesure des débits capillaires, ce qui indique qu'il est indispensable d'appliquer une étape de déacétylation pour des mesures réalisées au niveau hépatique chez des ovins. En revanche, les contributions publiées qui ont été obtenues avec la méthode de Katz et Bergman [27] sont en moyenne de $18 \%$ (7-27\%), résultat correspondant à 16 publications sur ovins $[3,4,6-10,12,13,20,26,27$, $33,34,39,47]$. Les contributions obtenues avec cette méthode confirment la nécessité d'ajouter de l'acide chlorhydrique pour la déacétylation et montrent qu'il faut impérativement vérifier la déacétylation complète du PAH par la méthode employée.

Toutefois, la méthode de dosage avec déacétylation présente une proportion plus importante de débits négatifs en artère hépatique, par rapport aux autres méthodes de dosage utilisées. Ces valeurs calculées ne sont pas physiologiquement valides, mais s'expliquent par les limites de détection du dosage déjà mentionnées auxquelles s'ajoute la variabilité physiologique des débits sanguins. Une variabilité intra-cycle de l'ordre de 12 à $16 \%$ (obtenue avec un nombre de 9 prélèvements par cycle alimentaire) permet de détecter des différences de débits instantanés de l'ordre de 18 à $24 \%$. Par conséquent, ces résultats suggèrent qu'il n'est pas possible de déterminer avec fiabilité les variations post-prandiales de débits sanguins splanchniques instantanés, tout particulièrement au niveau artère hépatique, lorsque celles-ci sont inférieures à $24 \%$. L'utilisation de cette méthode de mesure des débits sanguins à l'aide du PAH aux doses d'infusion considérées se limite à l'obtention de débits sanguins moyens, telle que préconisée par Katz et Bergman 
[27]. Dans ce cas, nous avons calculé qu'en moyenne les traitements doivent modifier les débits d'au moins $20 \%$ pour que l'effet soit détectable statistiquement avec des effectifs de quatre à cinq animaux par traitement. De tels effets sont généralement obtenus par des variations de niveau alimentaire [37], et non par des modifications de la nature du régime [24] ni par des perfusions de nutriments [42] ou d'hormones spécifiques [43].

\subsection{Conclusion}

La qualité des quatre méthodes de dosage du PAH testées ne présente pas de différences significatives. Il semble souhaitable d'effectuer une gamme dans du sang pour effacer les effets matrices. La déacétylation en présence d'acide chlorhydrique est essentielle lorsque l'on effectue des mesures au niveau du foie, car $13 \%$ du PAH infusé est acétylé au niveau du foie en moyenne. En revanche, lorsque l'on veut comparer des résultats de débits sanguins, il semble important de tenir compte de la méthode de dosage utilisée. En conclusion, l'utilisation de cette technique de dilution à l'aide du PAH se limite à l'obtention de débits moyens et paraît difficilement utilisable lorsque l'on veut effectuer des mesures de débit sanguin en cinétique.

\section{REMERCIEMENTS}

Les auteurs remercient J.-P. Chaise, C. Léoty et $\mathrm{R}$. Souchet pour leurs soins aux animaux, F. Duboisset et C. Legay pour leur aide aux prélèvements et $\mathrm{G}$. Bielicki pour les analyses de RMN.

\section{REFERENCES}

[1] Apatu R.S.K., Barnes R.J., Blood flow to and the metabolism of glucose and lactate by the liver in vivo in fetal, newborn and adult sheep, J. Physiol. 436 (1991) 431-447.

[2] Apatu R.S.K., Barnes R.J., Release of glucose from the liver of fetal and postnatal sheep by portal vein infusion of cathecholamines or glucagon, J. Physiol. 436 (1991) 449-468.

[3] Bergman E.N., Wolff J.E., Metabolism of volatile fatty acids by liver and portal-drained viscera in sheep, Am. J. Physiol. 221 (1971) 586-592.

[4] Bergman E.N., Katz M.L., Kaufman C.F., Quantitative aspects of hepatic and portal glucose metabolism and turnover in sheep, Am. J. Physiol. 219 (1970) 785-793.

[5] Bratton C.A., Marshall E.K., A new coupling component for sulfanil-amide determination, J. Biol. Chem. 128 (1939) 537-550.

[6] Brockman R.P., Role of insulin in regulating hepatic gluconeogenesis in sheep, Can. J. Physiol. Pharmacol. 63 (1985) 1460-1464.

[7] Brockman R.P., Effects of epinephrine on the net hepatic uptake of lactate, pyruvate and glycerol in sheep, Can. J. Physiol. Pharmacol. 69 (1991) 475-479.

[8] Brockman R.P., Bergman E.N., Effect of glucagon on plasma alanine and glutamine metabolism and hepatic gluconeogenesis in sheep, Am. J. Physiol. 228 (1975) 1627-1633.

[9] Brockman R.P., Bergman E.N., Quantitative aspects of insulin secretion and its hepatic and renal removal in sheep, Am. J. Physiol. 229 (1975) 1338-1343.

[10] Brockman R.P., Bergman E.N., Joo P.K., Manns J.G., Effects of glucagon and insulin on net hepatic metabolism of glucose precursors in sheep, Am. J. Physiol. 229 (1975) 1344-1350.

[11] Brown N.D., Lofberg R.T., Gibson T.P., A study of the Bratton and Marshall hydrolysis procedure utilizing high performance liquid chromatography, Clin. Chim. Acta 70 (1976) 239-245

[12] Burrin D.G., Ferrell C.L., Eisemann J.H., Britton R.A., Level of nutrition and splanchnic metabolite flux in young lambs, J. Anim. Sci. 69 (1991) 1082-1091.

[13] Burrin D.G., Ferrell C.L., Eisemann J.H., Britton R.A., Nienaber J.A., Effect of level of nutrition on splanchnic blood flow and oxygen consumption in sheep, Br. J. Nutr. 62 (1989) 23-34.

[14] Freetly H.C., Ferrell C.L., Kinetics of splanchnic progesterone metabolism in ewes fed two levels of nutrition, J. Anim. Sci. 72 (1994) 2107-2112.

[15] Freetly H.C., Ferrell C.L., Net uptakes of oestradiol-17 $\beta$ and progesterone across the portal-drained viscera and the liver of ewes, $J$. Endocrinol. 141 (1994) 353-358. 
[16] Freetly H.C., Ferrell C.L., Jenkins T.G., Goetsch A.L., Visceral oxygen consumption during chronic feed restriction and realimentation in sheep, J. Anim. Sci. 73 (1995) 843-852.

[17] Goetsch A.L., Ferrell C.L., Effects of dietary maize level on net flux across splanchnic tissues of oxygen and nutrients in wethers consuming ad libitum different forages, Anim. Sci. 61 (1995) 43-55.

[18] Goetsh A.L., Ferrell C.L., Freetly H.C., Effects of different supplements on splanchnic oxygen consumption and net fluxes of nutrients in sheep consuming bromegrass (Bromus inermis) hay ad libitum, Br. J. Nutr 72 (1994) 701-712.

[19] Hamburger J., Ryckewaert, Duizend, Argant, Microdosage de l'acide para-amino-hippurique dans le sang et dans l'urine, Ann. Biol. Clin. 6 (1948) 358-362.

[20] Harman N.G., Pethick D.W., The effects of sustained exercice on gluconeogenesis, glycogenolysis and glycogen synthesis in Merino sheep, Aust. J. Agric. Res. 45 (1994) 1189-1202.

[21] Harvey R.B., Brothers A.J., Renal extraction of para-aminohippurate and creatinine measured by continuous in vivo sampling of arterial and renal-vein blood, Ann. N.Y. Acad. Sci. 102 (1962) 46-54.

[22] Herdt T.H., Wensing T., Haagsman H.P., van Golde L.M.G., Breukink H.J., Hepatic triacylglycerol synthesis during a period of fatty liver development in sheep, J. Anim. Sci, 66 (1988) 1997-2013.

[23] Huntington GB (1982) Portal blood flow and net absorption of ammonia-nitrogen, and glucose in nonlactating Holstein cows, J. Dairy Sci. 65, 1155-1162.

[24] Huntington G.B., Hepatic urea synthesis and site and rate of urea removal from blood of beef steers fed alfalfa hay or a high concentrate diet. Can. J. Anim. Sci. 69 (1989) 215-223.

[25] Isserty A., Ortigues I., Méthodes d'exploitation de données concernant les débits sanguins mesurés au niveau des viscères et du train-arrière chez la brebis, Reprod. Nutr. Dev. 34 (1994) 399-413.

[26] Katz M.L., Bergman E.N., Hepatic and portal metabolism of glucose, free fatty acids and ketone bodies in the sheep, Am. J. Physiol. 216 (1969) 953-960.

[27] Katz M.L., Bergman E.N., Simultaneous measurements of hepatic and portal venous blood flow in the sheep and dog, Am. J. Physiol. 216 (1969) 946-952.

[28] Kirch E.R., Bergeim O., Determination of paminobenzoic acid, J. Biol. Chem. 148 (1943) $445-450$.
[29] Lobley G.E., Connel A., Lomax M.A., Brown D.S., Milne E., Calder A.G., Farningham D.A.H., Hepatic detoxification of ammonia in the ovine liver: possible consequences for amino acid catabolism, Br. J. Nutr. 73 (1995) 667-685.

[30] Lobley G.E., Connel A., Revell D.K., Bequette B.J., Brown D.S., Calder A.G., Splanchnic-bed transfers of amino acids in sheep blood and plasma, as monitored through use a multiple U-13C-labelled amino acid mixture, Br. J. Nutr. 75 (1996) 217-235.

[31] Lobley G.E., Weijs P.J.M., Connell A., Calder A.G., Brown, Milne E., The fate of absorbed and exogenous amonia as influenced by forage or forage-concentrate diets in growing sheep, Br. J. Nutr. 76 (1996) 231-248.

[32] Looyé A., Automated simultaneous determination of p-acetylaminohippurate and insuIin in serum, Clin. Chem. 16 (1970) 753-755.

[33] Mineo H., Yasuda T., Akiyama M., Oyamada T., Kato S., Ushijima J., Effect of feeding on hepatic and portal blood flow in sheep, Small Ruminant Res. 5 (1991) 181-186.

[34] Naylor J.M., Kronfeld D.S., Freeman D.E., Richardson D., Hepatic and extrahepatic lactate metabolism in sheep: effects of lactate loading and $\mathrm{pH}$, Am. J. Physiol. 247 (1984) E747-E755.

[35] Newman E., Kattus A., Genecin A., Genest J, Calkins E., Murphy J., Observations on the clearance method of determining renal plasma flow with diodrast, para-aminohippuric acid (PAH) and para-acetyl-aminohippuric acid (PACA), Bull. Johns Hopkins Hosp. 84 (1949) 135-168.

[36] Ortigues I., Visseiche A.L., Whole-body fuel selection in ruminants: nutrient supply and utilization by major tissues, Proc. Nutr. Soc. 54 (1995) 235-251.

[37] Ortigues I., Durand D., Lefaivre J., Use of para-amino-hippuric acid to measure blood flows through portal drained viscera, liver and hindquarters in sheep, J. Agric. Sci. Camb. 122 (1994) 299-308.

[38] Patil A.R., Goetsh A.L., Park K.K., Kouakou B., Galloway D.L., West C.P., Johnson Z.B., Net flux of nutrients across splanchnic tissues in sheep fed tropical vs. temperate grass hay of moderate or low qualities, Livest. Prod. Sci. 43 (1995) 49-61.

[39] Pethick D.W., Miller C.B., Harman N.G. Exercice in Merino sheep - the relationships between work intensity, endurance, anaerobic threshold and glucose metabolism, Aust. J. Agric. Res. 42 (1991) 599-620.

[40] Rémond D., Poncet C., Lefaivre J., Technical note: ruminal vein catheterization and continuous blood flow measurement in ruminal 
arteries of sheep, J. Anim. Sci. 71 (1993) 1276.

[41] Rémond D., Ortigues I., Isserty A., Lefaivre J., Technical note: Measuring portal blood flow in sheep using ultrasonic transit time flow probe, J. Anim. Sci. (sous presse).

[42] Reynolds C.K., Tyrrell H.F., Armentero L.E. Effects of mesenteric vein $n$-butyrate infusion on liver metabolism by beef steers, J. Anim. Sci. 70 (1992) 2250-2261.

[43] Reynolds C.K., Lapierre H., Tyrrell H.F., Elsasser T.H., Staples R.C., Gaudreau P., Brazeau P., Effects of growth hormone-releasing factor and feed intake on energy metabolism in growing beef steers: net nutrient metabolism by portal-drained viscera and liver, J. Anim. Sci. 70 (1992) 752-763.

[44] Smith H.W., Finkelstein N., Aliminosa L., Crawford B., Graber M., The renal clearances of substituted hippuric acid derivatives and other aromatic acids in dog and man, J. Clin. Invest. 24 (1945) 388-403.

[45] Sokal R.R., Rohlf F.J., Biometry, WH Freeman and Co, San Francisco, 1969.

[46] Technicon Industrial Systems, Para-aminohippuric acid, Technicon Industrial method,

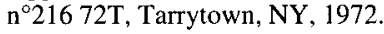

[47] Wolff J.E., Bergman E.N., Williams H.H. Net metabolism of plasma amino acids by liver and portal-drained viscera of fed sheep, Am. J. Physiol. 223 (1972) 438-446.

[48] Wray-Cahen D., Bell A.W., Boyd R.D., Ross D.A., Bauman D.E., Krich B.J., Harrell R.J., Nutrient uptake by the hindlimb of growing pigs treated with porcine somatotropin and insulin, J. Nutr. 125 (1995) 125-135. 